\title{
RECIST criteria and clinical practice
}

\author{
Piotr J. Wysocki \\ Oncology Chair and Clinic, Jagiellonian University, Collegium Medicum, Krakow, Poland
}

The objective of response evaluation criteria in solid tumours is to assess the reaction of cancer lesions to the applied treatment. Categorisation of the response to oncological treatment was proposed for the first time by the WHO in 1981 [1], however, over the subsequent two decades, no detailed or generally accepted guidelines were actually established. It was only in the mid-1990s that work on the standardisation of treatment response criteria began, and, in 2000, the first version of RECIST criteria was published [2]. The criteria were soon accepted by international regulatory agencies, such as the FDA or EMA. In 2009 the criteria were updated, making up RECIST 1.1 [3]; this version, with only some modifications connected with the introduction of immunotherapy [4], has defined the standards of objective evaluation of treatment response in oncology until today. In 2014 Nowotwory. Journal of Oncology published a paper introducing the RECIST 1.1 evaluation criteria to Polish readers [5].

The core issue that all oncologists must remember is the fact that RECIST criteria cannot assess whether patients will objectively benefit from treatment; instead it can verify if there was an objective reduction of the cancer stage in the cases of these patients. Thus, RECIST criteria, though very useful for the evaluation and comparison of new medication and treatment strategies with the standard ones, should not be the only decision criterion in oncological practice. What is more, in some cases such a means of treatment could turn out to be adverse for patients.

One can imagine a situation in which a patient with a massive and symptomatic cancer dissemination into the visceral organs, receives systemic treatment which allows for a clear imagining and clinical response within all the metastatic lesions. However, after a few months of disease control in the visceral organs, two new metastases appear in the bone system. In accordance with RECIST criteria, this means disease progression. Does it mean, though, that in a patient with a very good and permanent clinical response (symptom resolution, improvement of organ efficiency), effective systemic treatment must be discontinued because of two new asymptomatic lesions appearing? The answer is - no. Systemic treatment must be maintained as it comprehensively controls the disease whilst the introduction of local treatment must be taken into consideration, e.g. stereotactic radiotherapy which can get rid of asymptomatic progression in the bones. Such situations are quite frequent in clinical practice. This confirms only that the possibility of response evaluation according to RECIST criteria does not exempt oncologists from thinking and treating the patient's wellbeing, and not the size or number of cancer lesions, as the priority.

Drug programmes which we have at our disposal were created on the basis of clinical trial protocols so as to maximise the probability of reaching therapeutic effect compliant with the results of registration studies for a specific therapy. This is why many patients who do not meet the strictly defined inclusion criteria may not have access to new treatment methods. At the same time, following the study protocols is necessary for the reimbursement of extremely costly specific therapies. Qualification and treatment within the drug programmes requires

\section{How to cite:}

Wysocki PJ. RECIST criteria and clinical practice. NOWOTWORY J Oncol 2021; 71: 319-320. 
a good knowledge and interpretation of these provisions. This is to ensure that the chances of inclusion into the programmes of the patients who meet the detailed requirements of the payer can be increased.

The Polish Society of Clinical Oncology, in collaboration with marketing authorisation holders, carried out a number of courses and online conferences concerning drug programmes, regularly answering questions from doctors about the methods of interpretation of specific provisions in the regulations. The objective is to increase the chances of oncological patients in Poland getting access to the most novel strategies of systemic treatment which may potentially improve their prognoses. Thanks to this process, doubts concerning qualifications to the drug programmes without the changes measurable according to RECIST 1.1 criteria were finally resolved. At the same time, it was explained that the lesions which can be assessed are all the lesions (both target and non-target) visible in the imaging diagnostics which can be monitored both with regards to their size and also their number and morphology.

To sum up, it must be emphasised that RECIST criteria are of key importance first of all in clinical studies and, in some respect, also in the monitoring of patients treated within the drug programmes. In clinical practice in turn, they make up an additional source of information about the activity of various oncological therapies. They should not, however, exempt oncologists from clinical diligence and from ensuring the patient's wellbeing is the central point of the decision-making process.
Such an attitude is necessary for obtaining the best possible effect of the systemic oncological treatment applied - irrespective of whether it is carried out within the drug programmes or the therapy available in the catalogue.

Conflict of interests: not reported

\section{Piotr J. Wysocki}

Jagiellonian University

Collegium Medicum

Oncology Chair and Clinic

ul. Śniadeckich 10

31-531 Kraków, Poland

e-mail:piotr.wysocki@uj.edu.pl

Received and accepted: 31 Aug 2021

\section{References}

1. Miller $A B$, Hoogstraten $B$, Staquet $M$, et al. Reporting results of cancer treatment. Cancer. 1981; 47(1): 207-214, doi: 10.1002/ 1097-0142(19810101)47:1<207::aid-cncr2820470134>3.0.co;2-6.

2. Therasse $P$, Arbuck $S$, Eisenhauer $E$, et al. New Guidelines to Evaluate the Response to Treatment in Solid Tumors. J Natl Cancer Inst. 2000; 92(3): 205-216, doi: 10.1093/jnci/92.3.205.

3. Eisenhauer EA, Therasse P, Bogaerts J, et al. New response evaluation criteria in solid tumours: Revised RECIST guideline (version 1.1). Eur J Cancer. 2009; 45(2): 228-247, doi: 10.1016/j.ejca.2008.10.026.

4. Seymour L, Bogaerts J, Perrone A, et al. iRECIST: guidelines for response criteria for use in trials testing immunotherapeutics. The Lancet Oncology. 2017; 18(3): e143-e152, doi: 10.1016/s1470-2045(17)30074-8.

5. Płużański A. Evaluation of response to treatment — criteria RECIST 1.1. Nowotwory. Journal of Oncology. 2014; 64(4): 331-335, doi: 10.5603/ njo.2014.0055 